\title{
Research Review of the Ecological Carrying Capacity
}

\author{
Xilian Wang \\ College of Management, Xi'an University of Science and Technology, Xi'an 710054, China
}

\begin{abstract}
Supported by the Educative Foundation of Xi'an University of Science and Technology (Project Number:
\end{abstract} 2009041)

\begin{abstract}
The ecological carrying capacity is of vital importance for the economic development and the scale of resource exploitation in one country or region. This paper more comprehensively summarizes and concludes the basic contents and evaluation methods of ecological carrying capacity with regard to the related studies of the domestic and foreign scholars.
\end{abstract}

Keywords: Ecology, Carrying capacity, Sustainable development

\section{The origin of carrying capacity}

From the industrial revolution in the latter half of $18^{\text {th }}$ century, the material civilization of human beings is developed highly in the process of exploiting and making use of natural resources. At the same time of immersing in the huge material achievement brought by the industrial civilization, the human beings are suffering a serious of important problems: the pollution of air and water, soil loss, desertification becoming larger, climate changing abnormally, biological species decreasing quickly, the shortage or exhaustion of resources, the destruction of ozone layer, population increasing sharply and so on. The traditional economic development mode and limited resource environment capacity require the resources exploitation to be controlled in the ecological carrying capacity, in that the sustainable development of social economy can be achieved by the resources being used continuously. In 1921, Park and Burgess proposed the concept of carrying capacity and held the opinion that the carrying capacity of population in one region can be determined according to food resources in the region; with the problems of land degradation, environmental pollution, resources exhaustion and population expansion, the biologists and ecologists develop the concept of carrying capacity and apply it to human ecology etc, and the concepts of carrying capacity about soil resources, water resources, forest resources and environment are proposed one after the other. To sum up, from the concept of carrying capacity being proposed to now, it has changed profoundly in the form and significance. The evolvement and development of the concept of carrying capacity is displayed in table 1.

\section{The concept and connotation of ecological carrying capacity}

Another problem is ecological destruction that cannot be divided from resources shortage and environment pollution. Ecological destruction may damage the integrality of ecosystem, in that the human beings and different kinds of animals which survive in the ecological system may face the survival crisis. Therefore, many scholars and experts proposed to preserve the integrality of ecosystem and control the activities of human beings in the capacity of ecosystem, in that ecological carrying capacity arises. The referred foreign research reports define the ecological carrying capacity mostly in the view of species ecology. (Bailey J.A. etc, 1984) divided the carrying capacity into "economic carrying capacity" and "ecological carrying capacity", among them the ecological capacity belongs to the category of wildlife management which refers to the equilibrium of species and environment without the interference from hunting and so on, that means the ecological carrying capacity are decided by the biological resources in the condition that hunting does not exist or the normal hunting has little influence on the number of species; (A.C.smaal, T.c.Prins et, 1998) consider that the ecological carrying capacity refers the maximum of species which are supported by the special ecosystem in the special time; (Andrew, T. Hudak (1999) think that the ecological carrying capacity refers the maximum of species which are supplied by the vegetations in the special time. It can be concluded the connotation of the ecological carrying capacity, which refers the maximum of species held by the ecosystem in the existing condition.

Chinese researchers did research on the ecological carrying capacity in 1990s, which is still in the starting stage. (Jiaji, Wang, 2000) considered that the ecological carrying capacity is the objective reflection of the adjusting ability of natural system; (Jixi, Gao, 2001) considered that the ecological carrying capacity is the capacity of self-maintenance and self-regulation, the accommodating ability of resources and environment subsystem and the strength of social and economic activities and the number of people living normally sustained by the 
subsystem. He also points out the basic conditions, the constraint conditions and the supporting conditions of the ecological carrying capacity. Some other scholars point out the essence of the ecological carrying capacity in the view of systemic conformity, which means that the ecological carrying capacity is manifested as two aspects including the capability of ecological environment and the self-recovery ability of system, which in fact reflects the harmonious, interactive, symbiotic relationship between people and ecosystem.

\section{Evaluating methods for the ecological carrying capacity}

The theory about the ecological carrying capacity has become mature gradually, which developed from the initially qualitative static research to the quantitative dynamic prediction. The evaluating models used for the ecological carrying capacity at home and abroad mainly include the natural Vegetation NPP method, the balance law of supply and demand, the state space method, the ecological footprint method, comprehensive evaluation methods and so on.

The Vegetation NPP refers to the amount of organic compounds accumulated by vegetations in unit of time and area, which reflects the productive capacity of vegetation community in the natural environment. NNP is an important index for evaluating the framework and functional features of ecosystem and the capacity of ecosphere for carrying human beings, which is also an important index for estimating the supporting ability of the earth and evaluating the continuous development of the ecosystem in land. In 1975, Lieth, and the subsequent Ulittaker, Uchijima etc, considering about emphasizing particularly on all kinds of regulating factors and understanding the regulation mechanism differently according to the difficult degree of molds, divide the NPP molds to three species: climate statistical mold, process mold and light energy utilization molds.

The regional ecological carrying capacity reflects the degree of the ecosystem satisfying the development of regional society and economy and all the requirement of people in the aspect of all kinds of resources and the quality of ecological environments. To simplify the analytical calculation of the ecological carrying capacity and strengthen the maneuverability, Zhonggen Wang, Jun Xia etc proposed a measuring method based on the supply-demand relationship, namely the balance law of supply and demand. This method need to build a set of index system, including the indexes of socioeconomic system and environmental system (involving environment resources and quality).

The state space method describes external features, internal states and features of the system, which is an effective method to quantitatively describe the system state using the euclidean geometry space. The state space axis, in three dimensions, of ecological carrying capacity separately represent the number of human beings, the economic and social activities, the regional resources environment, the carrying state point can denote the carrying condition of one region in a period of time. The carrying state points form to carrying surfaces. The points above the carrying surface denote over loading and the ones under the surface denote deficient loading and the ones just on the surface denote full loading.

The Canadian ecological economic experts E. R. William and M. Wackernagel proposed the concept of the ecological footprint method, which evaluates the effect that people impose on the ecosystem through measuring the natural amount used for the survival of human beings and introduced the concept of ecological productive area to quantified the human beings on the demand for the regional ecosystem and the carrying capacity of the regional ecosystem, two of which can be compared to quantified the extent of regional development to afford a new method for quantitatively measuring the degree of continuing development.

Jixi Gao exploringly proposed the mold and method for measuring the ecological carrying capacity and pointed that the ecological carrying capacity can adopt the method of classification evaluation: the degree of the ecological elasticity may be used as the criteria for the first evaluation and the condition of resources and environment can be regarded as the criteria for the second evaluation and the carrying pressure can be used as the criteria for the third evaluation. He also did some research on the ecological carrying capacity and the continual development in the basin of Black River. Youpeng Xu made multiple-factor evaluation on the carrying capacity through comprehensive judgment matrix on the basis of evaluating each factor affecting the carrying capacity of water resources by using fuzzy comprehensive estimation. He also built a set of index system and calculation method which is suitable for comprehensively estimating the carrying capacity of oasis water resources.

Compared with the five methods comprehensively, the natural Vegetation NPP method is feasible but it doesn't reflect all kinds of human beings' social economic activity capacity that the ecological environment endures; the balance law of supply and demand is clear and simple but it doesn't reflect the socioeconomic status and the people's living standards in the area; the state space method more accurately estimates the condition of carrying capacity in certain period of time in certain area, but it is more difficult to calculate and build curved surface of 
bearing capacity and also need more materials; with great advantage in theory and application, the ecological footprint method has its unique aspects in assessing human beings' impact on ecological system, but the method, based on static analysis and ecological bias, only emphasizes regional ecological sustainable development but doesn't further judge the sustainable development of regional society and economy sectors; comprehensive evaluation judging modes and methods of ecological carrying capacity is relatively better than the other four methods, which combines grade assessment with comprehensive assessment, making the result clearer, more accurate and effective, and is also beneficial to the regulation and control of sustainable development, but the method, on the one hand, has strict requirements in collecting and dealing with data, and the determination of score and weight has certain arbitrariness and subjectivity on the other hand, it needs more data information.

\section{References}

Andrew, Hudak T. (1999). Rangeland Mismanagement in South Africa: Failure to Apply Ecological Knowledge. Human Ecology, 1999, 27(1):55 78.

Boyden,S., Dovers, S. (1992). Nature-resource consumption and its environment impacts in the western world: impacts of increasing Per capita consumption. Ambio, 1992, 21(1): 63 69.

Cohen, J. E. (1995). Population growth and earth's human carrying capacity. Science, 1995, 269:341 346.

Daily G C, Ehrlich P R. (1992). Population, sustainability, and earth's carrying capacity. Bioscience, 1992, 42(10):761 771.

David Price. (1999). Carrying Capacity Reconsidered. Population and Environment, 1999, 21(1):5 26.

Peng, Zaide. (1996). Preliminary exploration on the methods of the carrying capacity of regional environment. China Environmental Science, 1996, 6(1):6 10.

Wang, Honggen \& Xia, Jun. (1999). Research on quantification approach to the carrying capacity of regional ecological environment. Journal of Yangtze River University of Staff and Workers, 1999,16(4):9 12.

Wang, Jiaji, Yao, Xiaohong \& Li, Jingrong. (2000). Estimation of the ecological carrying capacity in in the Heihe River Basin. Research of Environmental Sciences, 2000, 13(2):44 48.

Table 1 . The research field and connotation of carrying capacity

\begin{tabular}{ccc}
\hline name & $\begin{array}{c}\text { Development } \\
\text { background }\end{array}$ & Basic connotation \\
\hline $\begin{array}{c}\text { Specious } \\
\text { carrying } \\
\text { capacity }\end{array}$ & $\begin{array}{c}\text { Ecology } \\
\text { development }\end{array}$ & The carrying quantity of other species which live in the ecology \\
system
\end{tabular}

